\title{
Sensor Selection Heuristic in Sensor Networks
}

\author{
Vaishali P. Sadaphal, Bijendra N. Jain \\ Department of Computer Science and Engineering \\ Indian Institute of Technology Delhi \\ Hauz Khas, New Delhi 110016, India \\ \{vaishali,bnj\}@ese.iitd.ernet.in
}

\begin{abstract}
We consider the problem of estimating the location of a moving target in a 2-D plane. In this paper, we focus attention on selecting an appropriate $3^{\text {rd }}$ sensor, given two sensors, with a view to minimize the estimation error. Only the selected sensors need to measure distance to the target and communicate the same to the central "tracker". This minimizes bandwidth and energy consumed in measurement and communication while achieving near minimum estimation error. In this paper, we have proposed that the $3^{\text {rd }}$ sensor be selected based on three measures viz. (a) collinearity, (b) deviation from the ideal direction in which the sensor should be selected, and (c) proximity of the sensor from the target. We assume that the measurements are subject to multiplicative error. Further, we use least square error estimation technique to estimate the target location. Simulation results show that using the proposed algorithm it is possible to achieve near minimum error in target location.
\end{abstract}

\section{Introduction}

We consider the problem of estimating the location of a moving target ' $\mathrm{T}$ ' in a 2-D plane. The target is moving at varying speed and direction (see Figure 1). For the present, we assume that the target is not aware of its own location. Or, if it is aware of its location, then it does not share this information with any other device. In either case, we assume that it is possible for sensors, such as $s^{1}$ located at $\left[x_{1}, y_{1}\right]$, to "measure" the distance from/to the target, located at $\left(x_{0}, y_{0}\right)$, and thereby estimate the location of target ' $T$ '. Several methods for measuring distance between a sensor and the target are available. See [1], [2] for methods based on radio signal strength (RSSI) and [3], [4] for methods based on time difference of arrival (TDOA).

Irrespective of the method used to measure distance, all such measurements are subject to error. Two models have been studied, viz. (a) additive, and (b) multiplicative. In this paper, we confine ourselves to using the multiplicative model for errors. Let $d_{A}^{i}$ and $d_{M}^{i}$ be the actual and measured distance between target and the $i^{t h}$ sensor. The multiplicative error in measurement by $i^{\text {th }}$ sensor, $e_{m}^{i}=d_{A}^{i}-d_{M}^{i}=\gamma^{\frac{\varepsilon_{\text {meas }}}{2}} d_{A}^{i}$ where, $\varepsilon_{\text {meas }}$ is a measure of the amount of measurement error, and $-1 \leq \gamma \leq 1$. That is, the error is uniformly distributed with endpoints $\left(1-\frac{\varepsilon_{\text {meas }}}{2}\right) d_{A}^{i}$ and $\left(1+\frac{\varepsilon_{\text {meas }}}{2}\right) d_{A}^{i}$, zero mean and standard deviation of $\sigma_{m}^{i}=\frac{1}{\sqrt{3}}\left(\frac{\varepsilon_{\text {meas }}}{2}\right) d_{A}^{i}$.

Even though the distance between a given sensor and the target is known only with some error, it is possible to use distance measurements from 3 or more sensors to 'estimate' the location of the target assuming that (a) the location of the sensors is known to 
the central device responsible for estimating the location of the target, (b) the clocks of the sensors are synchronized so that the sensors "measure" distance at approximately the same time, and (c) the sensors are able to communicate their measurements to this central device, also referred to as the 'tracker".

Since the target is moving and since a sensor must be within a certain distance from the target (before it can detect the presence of the target and measure distance), we assume that there are several sensors, $\left\{s^{i}\right\}=\Sigma$, spread across the 2-D plane. In fact, we assume that there are three or more sensors located in and around every point in the 2-D plane so that we can compute an estimate based on measurements from a subset of three sensors suitably selected to minimize estimation error. This approach also allows one to minimize communication overheads and conserve battery power available to sensors. Further, since the target is moving, the collection of sensors changes every time an estimate is required to be obtained. Specifically, we assume that as the target moves, if sensors $\left\{s^{1}, s^{2}, s^{3}\right\}$ have made measurements at time $t_{k}$, then at time $t_{k+1}$, we drop one of the sensors $s^{1}, s^{2}$, or $s^{3}$ and select a sensor $s^{4}$ suitably so as to minimize the error in estimated location of the target. Accordingly, this paper is about suitably selecting the $3^{\text {rd }}$ sensor from a set of $N_{k+1}$ sensors.

\section{Mobile Target Tracking}

We now outline the overall scheme for tracking the target as it moves in the 2-D plane.

Let $L_{k}=\left[x_{k}, y_{k}\right]$ be the actual location of the target at time $t_{k}$. Let $\hat{L}_{k-1}=\left[\hat{x}_{k-1}, \hat{y}_{k-1}\right]$ and $\hat{L}_{k}=\left[\hat{x}_{k}, \hat{y}_{k}\right]$ be the estimated location of the target at time $t_{k-1}$ and $t_{k}$, respectively (see Figure 2). The latter estimate $\hat{L}_{k}$ is obtained using (a) an a-priori estimate of the target's location $\bar{L}_{k}=\left[\bar{x}_{k}, \bar{y}_{k}\right]$, and (b) measurements made at $t_{k}$ by sensors $\sigma_{k}^{1}, \sigma_{k}^{2}$, and $\sigma_{k}^{3}$ located at $\lambda_{k}^{1}, \lambda_{k}^{2}$, and $\lambda_{k}^{3}$, respectively. The a-priori estimate $\bar{L}_{k}=\left[\bar{x}_{k}, \bar{y}_{k}\right]$ may be based on its estimated location at time instants $t_{k-1}$ and $t_{k-2}$. (see Figure 2).

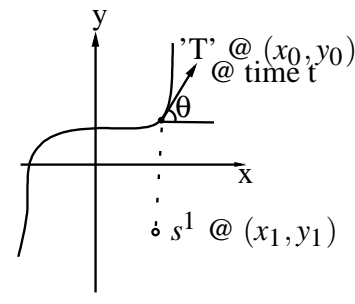

Fig. 1. Trajectory of the target ' $T$ ' in 2-D plane.

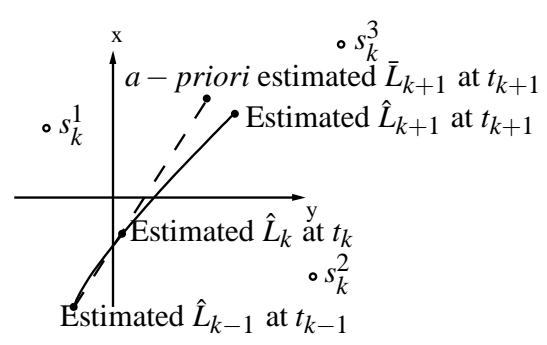

Fig. 2. Estimated location of the target on the basis of a-priori estimate.

The new estimate of the location of the target at time $t_{k+1}$ is obtained on the basis of new distance measurements $d_{k+1}^{1}, d_{k+1}^{2}$ from $\sigma_{k+1}^{1}, \sigma_{k+1}^{2}$ respectively, and $d_{k+1}^{3}$ from a newly selected sensor $\sigma_{k+1}^{3}$. Sensor $\sigma_{k+1}^{3}$ is selected assuming knowledge of a-priori estimate of the target location. The latter is an extrapolation of its location assuming 
that the average velocity during $\left[t_{k}, t_{k+1}\right]$ is the same as the average velocity during and $\left[t_{k}-1, t_{k}\right]$. The new estimate of the location of the target at time $t_{k+1}$ is obtained thus:

- Step 1: Given the estimated location $\hat{L}_{k}$ (based on measurements from $\sigma_{k}^{1}, \sigma_{k}^{2}$, and $\sigma_{k}^{3}$ ) and $\hat{L}_{k-1}$ at times $t_{k}$ and $t_{k-1}$, respectively, compute an a-priori estimate

$$
\bar{L}_{k+1}=\alpha_{k+1}\left(\hat{L}_{k}-\hat{L}_{k-1}\right)
$$

where, $\alpha_{k+1}=\frac{t_{k+1}-t_{k}}{t_{k}-t_{k-1}}$. (See Figure 2.)

- Step 2: Given its approximate location $\bar{L}_{k+1}$, identify an appropriate subset of three sensors, viz. $\sigma_{k+1}^{1}, \sigma_{k+1}^{2}$, and $\sigma_{k+1}^{3}$ from a given subset of sensors $\Sigma_{k+1} \subset \Sigma$.

- Step 3: Sensors $\sigma_{k+1}^{1}, \sigma_{k+1}^{2}$, and $\sigma_{k+1}^{3}$ obtain distance measurements $d_{k+1}^{1}, d_{k+1}^{2}$, and $d_{k+1}^{3}$, respectively, and communicate the same to the central 'tracker".

- Step 4: The 'tracker"computes a least square error estimate, $\hat{L}_{k+1}$, such that $\sum_{i=1}^{i=3} e_{i}^{2}$ is minimized. Here, $e_{i}=\left\|\hat{L}_{k+1}-\lambda_{k+1}^{i}\right\|^{\frac{1}{2}}-d_{k+1}^{i}$, where $\left\|\hat{L}_{k+1}-\lambda_{k+1}^{i}\right\|$ is the Euclidean distance between $\hat{L}_{k+1}$ and $\lambda_{k+1}^{i}=\left[x_{k+1}^{i}, y_{k+1}^{i}\right]$, the location of sensor $\sigma_{k+1}^{i}$. This method is described in Section 4.

In Step 2, we stipulate that sensors $\sigma_{k+1}^{i}$ are selected as follows: $\sigma_{k+1}^{1}=\sigma_{k}^{i}, j=1,2$, or $3 ; \sigma_{k+1}^{2}=\sigma_{k}^{i}, j=1,2$, or $3 ; \sigma_{k+1}^{2} \neq \sigma_{k+1}^{1}, \sigma_{k+1}^{3} \in \Sigma_{k+1} ; \sigma_{k+1}^{3} \neq \sigma_{k+1}^{1}, \sigma_{k+1}^{3} \neq \sigma_{k+1}^{2}$. That is, one of the sensors used to estimate the location of the target at time $k$ is replaced by another more suitable sensor from amongst the remaining set of sensors that are within the range of the target (see Sections 5 and 6).

Section 3 describes related work in the area of target tracking and sensor selection. Location estimation has been described in Section 4, while Section 5 and Section 6 describe the method and the algorithm for sensor selection, respectively. We present simulation results in Section 7. Section 8 concludes the paper.

\section{Related Work}

Research on estimating the location of a fixed target, given measurements from a subset of sensors has been reported in [1], [3], [5], [6], [7], [8], [9], [10], and [11]. These differ from each other on the basis of (a) the number of sensors required, (b) the nature of measurements, and (c) the technique for estimating the location.

- Priyantha et al [3] estimate target location using trilateration using distance measurements based on TDOA from 3 different sensors.

- Bahl et al [1] estimate target location using trilateration, but using RSSI measurements. Additionally, they build a radio map of the site and then locate the target based on radio signal strength measurements.

- Triangulation is used in robotics [11] to estimate the location of a robot. This requires 3 or more angle of arrival (AoA) measurements.

Tracking of mobile targets using sensor networks has been studied in [12], [13], [14]. 
- In [12] and [13] the target location is approximated by the location of a sensor when the target comes within its range. The resulting resolution is the same as that of the sensors. This may be, however, improved using multiple sensors.

- In [14] acoustic measurements from all sensors in the cluster around the target are used by a cluster head to minimize the error in estimating the location.

In this paper, and as described earlier in Section 2, a group of sensors in and around an a-priori estimated location of the target is selected. This group changes as and how the target moves within the 2-D plane.

Several papers (see [6], [7], [9], and [10]) have used different approaches to study selection of sensors.

- Zhao et al have proposed in [6] and [7] that sensors should be selected such that (a) communication overhead is minimized, and (b) the error in locating the target is minimized using a Bayesian maximum likelihood estimator. However, this assumes that an a-priori estimate of the location is available.

- Wang et al [9] also assume a-priori knowledge of the target location, expressed in the form of a Gaussian probability distribution function. The error in TDOA or AoA measurements is assumed to be Gaussian. While the estimate is based upon Bayesian filtering, the sensors selected are those that maximize the entropy difference between the a-priori and posteriori estimates of the target location.

- In Isler et al [10] the target location is estimated based on the region of intersection of 2-D cones resulting from uncertainty in AoA measurements from multiple sensors. Sensors which minimize the area of such intersection are the one selected. This scheme also requires an a-priori knowledge of the location of the target.

In this paper, we have proposed that a $3^{\text {rd }}$ sensor be selected given two sensors based on three measures viz. (a) collinearity, (b) deviation from the ideal direction, and (c) proximity of the sensor from the target. We use least square error estimation technique to estimate the target location.

\section{Target Location Estimation}

Before discussing selection of the $3^{\text {rd }}$ sensor, we discuss the method to obtain a least square error estimate of the location at time $t_{k}$. For convenience, we drop the subscript $k$ in $t_{k}$ and instead work with time $t$. The estimation problem can be stated thus: given measurements $d^{1}, d^{2}, \ldots, d^{n}$ from sensors $s^{1}, s^{2}, \ldots, s^{n}$, respectively located at $\lambda^{1}=$ $\left[x^{1}, y^{1}\right], \lambda^{2}=\left[x^{2}, y^{2}\right], \ldots, \lambda^{n}=\left[x^{n}, y^{n}\right]$, compute an estimate $\hat{L}=[\hat{x}, \hat{y}]$ such that

$$
\sum_{i=1}^{i=n}\left\{\sqrt{\left(\hat{x}-x^{i}\right)^{2}+\left(\hat{y}-y^{i}\right)^{2}}-d^{i}\right\}^{2}
$$

is minimum. The measurements are possibly subject to errors.

In order to compute the optimal $[\hat{x}, \hat{y}]$, we have experimented with two algorithms: (a) Steepest Descent algorithm [15], and with (b) Levenberg Marquardt algorithm [15] 
to compute the optimal $[\hat{x}, \hat{y}]$. In either case, the method requires an initial "guess". We have proposed that the initial estimate be obtained by solving $\left(\begin{array}{l}n \\ 2\end{array}\right)$ linear equations ${ }^{1}$.

Our experience (see also [16]) shows that (a) the above method for computing the initial guess is reasonably adequate in helping one to descend to the optimum (see Figure 3), (b) the error in location estimation increases (almost) linearly with error in measurement (see Figure 4), and (c) the number of iterations required in Steepest Descent method is generally less than 20, while the same is less than 6 in case of Levenberg Marquardt. In this paper, however, we use the Steepest Descent algorithm in all our simulations. (See Figure 3.)

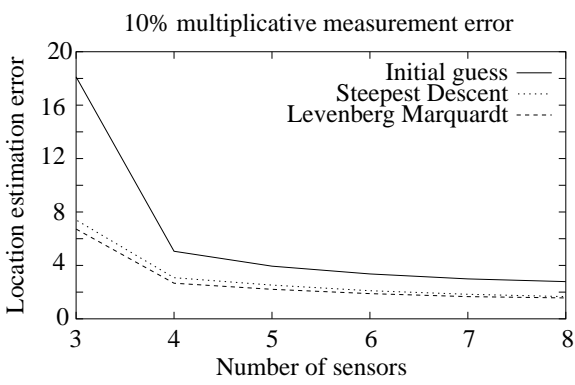

Fig. 3. Location estimation error in initial guess and in optimized result.

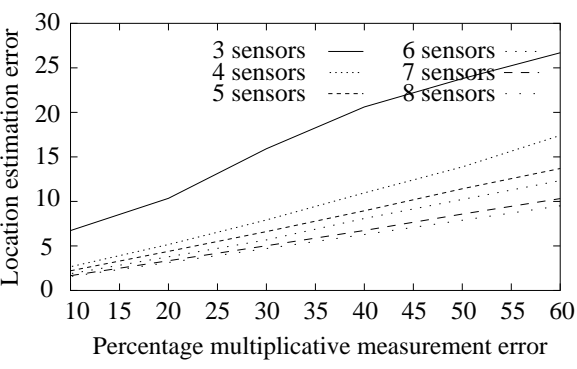

Fig. 4. Measurement error vs. Location estimation error.

\section{Sensor Selection}

The position of the $3^{\text {rd }}$ sensor relative to that of the other two sensors and the target plays an important role in location estimation accuracy. In a simulation, the $3^{r d}$ sensor, $s^{3}$, was placed at various locations and the resulting error in location estimation was computed. The sensing range, $r_{0}$, was assumed to be $100 \mathrm{~m}$. The measurement error was assumed to be $30 \%$, that is $\varepsilon_{\text {meas }}=0.3$. The target was assumed to be at $[0,0]$. The two sensor, $s^{1}$ and $s^{2}$, are placed at different positions such that (a) the visual angle made by them with the target, $\alpha>\frac{\pi}{2}$ or $\alpha<\frac{\pi}{2}$, and (b) $d^{1}=d^{2}$ or $d^{1} \neq d^{2}$.

Part of the results ${ }^{2}$ are given in Figures 5 and 6. It can be seen that (a) when sensors $s^{1}, s^{2}$, and $s^{3}$ are almost collinear, the error in estimated location is large, (b) there is a

\footnotetext{
${ }^{1}$ These are obtained by subtracting equations of the type $\sqrt{(\hat{x}-\dot{x})^{2}+(\hat{y}-\dot{y})^{2}}-d^{i}=0$ from one another, thereby resulting in $\left(\begin{array}{l}n \\ 2\end{array}\right)$ linear equations.

${ }^{2}$ A different symbol is printed for each range of estimation error. In particular, if $\mu$ is the average estimated error and $\varepsilon_{l o c}$ is the estimation error when the third sensor is placed in the specific position, then
}

- if $\varepsilon_{l o c} \geq \frac{3}{2} \mu$, then print "**, else

- if $\mu \leq \varepsilon_{\text {loc }}<\frac{3}{2} \mu$, then print " $\triangle$ ", else

- if $\frac{1}{2} \mu \leq \varepsilon_{\text {loc }}<\mu$, then print " +", else

- if $\varepsilon_{l o c} \leq \frac{1}{2} \mu$, then print "." 
preferred region in which to locate the $3^{\text {rd }}$ sensor. This is the region where a dot, "'.' is printed.

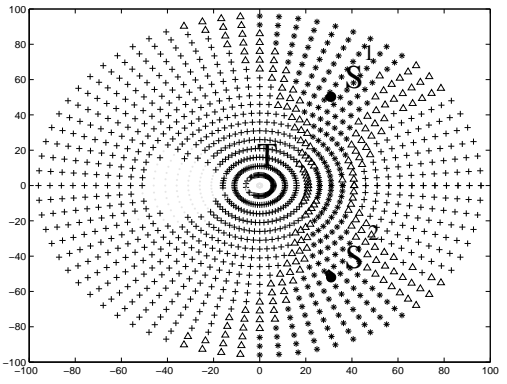

Fig. 5. $d^{1}=d^{2}, \alpha>\frac{\pi}{2}$.

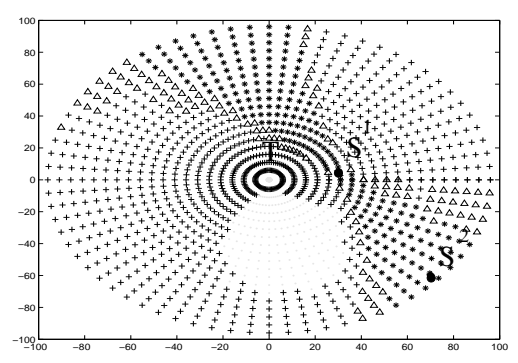

Fig. 6. $d^{1} \neq d^{2}, \alpha<\frac{\pi}{2}$.

The sensor selection technique proposed in this paper is based on three factors, viz. (a) collinearity of sensors, (b) ideal direction in which $3^{\text {rd }}$ sensor should be selected (for the given position of two sensors), and (c) proximity of selected sensor from the target. This selection is done before any new measurement is made, and assuming that the target is indeed located near the a-priori estimate of its location.

\subsection{Collinearity}

Consider the distribution of sensors $s^{1}, s^{2}$, and $s^{3}$ in a 2-D plane shown in Figure 7. If, for the moment, we assume that the error in distance measurements in near zero, then it can be concluded that the target is either located at position A, or at position B. Further, measurement from a third sensor does not add value since $s^{1}, s^{2}$ and $s^{3}$ are collinear. Distance measurement from a $3^{\text {rd }}$ sensor which is not collinear is necessary to resolve whether the target is at A or B.

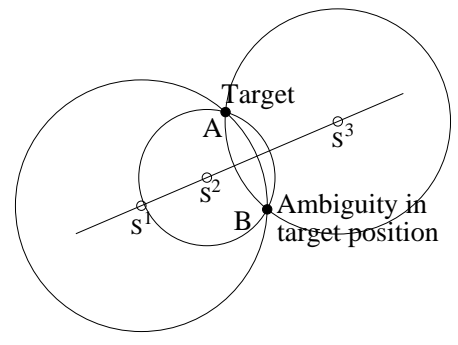

Fig. 7. Ambiguity in target location in case of collinear sensors.

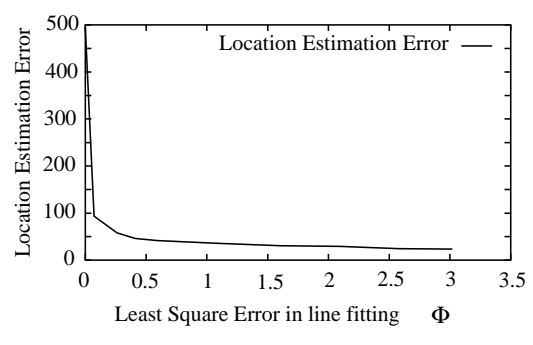

Fig. 8. Effect of collinearity on estimation error.

We define the collinearity coefficient $\Phi$ for an sensor $s^{i}$ as the residual error resulting from a linear least square fit through the given two sensors and the sensor $s^{i}$ : 


$$
\Phi\left(s^{1}, s^{2}, s^{i}\right)=\min _{(m, c)}\left\{\left(y^{1}-m x^{1}-c\right)^{2}+\left(y^{2}-m x^{2}-c\right)^{2}+\left(y^{i}-m x^{i}-c\right)^{2}\right\}
$$

Figure 8 shows a plot of estimation error vs. $\Phi$, the residual. When $\Phi$ is small, the sensors are almost collinear and the estimation error is high. But if the collinearity coefficient is large, the location estimation error is likely to be small.

\subsection{Ideal Direction}

Below we argue that, given the positions of two sensors, there is a preferred direction in which to locate the $3^{r d}$ sensor. Consider the region of intersection of the error annuli corresponding to the computed distance between the a-priori estimated location of the target and the two sensors (see Figure 9). Clearly, the region formed around the a-priori estimate of its location is the one in which the probability that the target is located is the maximum. For simplicity, this latter region is approximated by the parallelogram

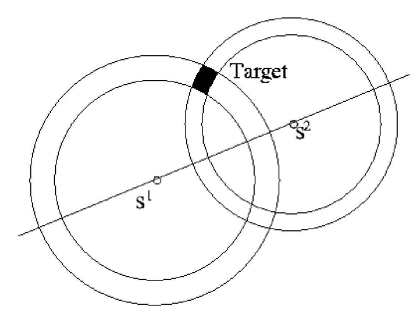

Fig. 9. Region of intersection of the error annuli.

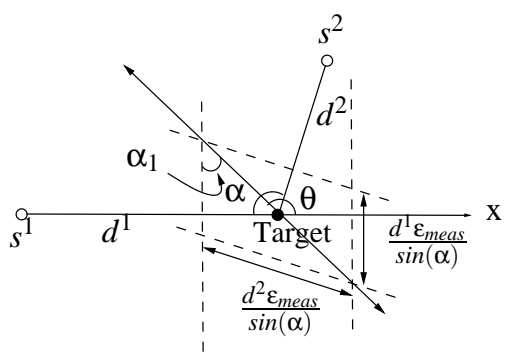

Fig. 10. Ideal direction in case $\alpha>\frac{\pi}{2}$.

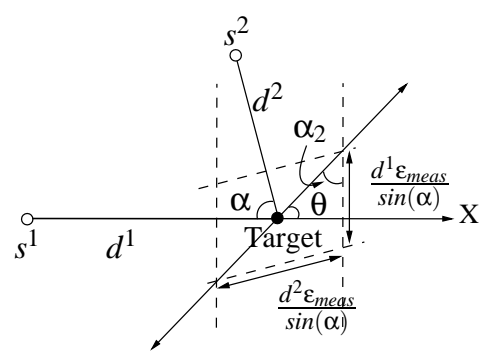

Fig. 11. Ideal direction in case $\alpha<\frac{\pi}{2}$.

obtained by intersecting bands formed by tangents to the error annuli. This is shown in Figures 10 and 11 for the two cases viz. (a) $\alpha>\frac{\pi}{2}$ and (b) $\alpha<\frac{\pi}{2}$, respectively. Here $\alpha$ is the visual angle made by the two sensors with the target. Irrespective of whether 
$\alpha>\frac{\pi}{2}$ or $\alpha<\frac{\pi}{2}$, we propose that the $3^{r d}$ sensor be placed along the larger diagonal of the parallelogram, since the resulting maximum uncertainty in the location of the target is minimized. This is discussed in detail below.

The ideal direction (see Figures 10 and 11), specified with respect to the axis passing from sensor $s^{1}$ to the target, is given by

$$
\begin{aligned}
& \theta=\frac{3 \pi}{2}+\tan ^{-1} \frac{d^{2} \sin (\alpha)}{d^{1}+d^{2} \cos (\alpha)}, \text { if } \alpha>\frac{\pi}{2}, \text { and } \\
& \theta=\frac{3 \pi}{2}+\alpha-\tan ^{-1} \frac{d^{1} \sin (\alpha)}{d^{2}-d^{1} \cos (\alpha)}, \text { if } \alpha<\frac{\pi}{2} .
\end{aligned}
$$

Selecting a sensor located approximately in the direction $\theta$ ensures that the resulting polygon of intersection after inclusion of measurement will have a smaller longest axis. A measure which captures the deviation from the ideal direction, and which may be used to select the $3^{\text {rd }}$ sensor, is defined thus:

$$
\Psi=\left|\theta_{s^{i}}-\theta\right|
$$

where $\theta_{s^{i}}$ is the direction of sensor $s^{i}$ with respect to the axis from $s^{1}$ to the target.

\subsection{Proximity of sensors to target}

We now argue that the $3^{\text {rd }}$ sensor is preferably placed as close as possible to the target. This is so only if measurement error is multiplicative.

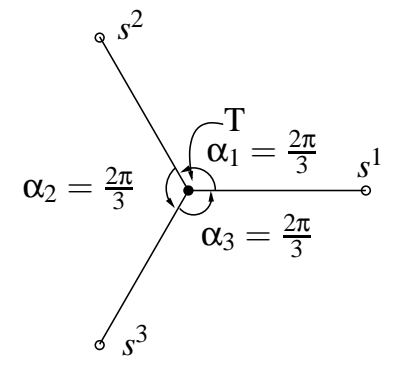

Fig. 12. Three sensors with equal visual angles $\alpha$.

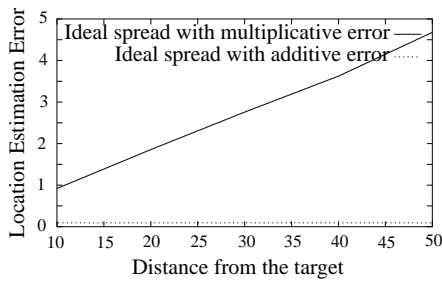

Fig. 13. Effect of distance on location estimation error.

From simulations (see Figure 13), it has been observed that the location estimation error increases almost linearly with the distance of the three sensors from the target. Since we have assumed a multiplicative error model, a measure of proximity $\Delta$ of sensor $s^{i}$ from the target is defined as:

$$
\Delta\left(s^{i}\right)=\left\|s^{i}-L\right\|,
$$

where $\left\|s^{i}-L\right\|$ is the Euclidean distance between $s^{i}$ and $L$. Since the location of the $\operatorname{target} L$ is unknown, we use $\bar{L}$, the a-priori estimate of the location.

$$
\Delta\left(s^{i}\right)=\left\|s^{i}-\bar{L}\right\|
$$




\section{Multi-objective Sensor Selection Algorithm}

In this paper, we propose that for given sensors $s^{1}$ and $s^{2}$, a $3^{r d}$ sensor $s^{3}$ is selected, from those sensors that are within the range of the target, have adequate battery power, and such that (a) the coefficient of collinearity is maximized, (b) the deviation of the direction of the sensor from the ideal direction is minimized, and (c) the distance of the sensor from the target is minimized. Since this is a multi-objective optimization problem, we propose the following algorithm:

- Step 1: Eliminate all sensors for which the collinearity coefficient $\Phi \leq \phi_{0}$.

- Step 2: Of the remaining sensors, consider only those for which deviation from the ideal direction $\Psi \leq \psi_{0}$.

- Step 3: Finally, of the remaining sensors, select the one for which the measure of proximity $\Delta$ is the minimum.

\section{Simulation Results}

Simulations were carried out with $N$ sensors $(\mathrm{N}=10,20$, or 30$)$, each of which is randomly placed within $100 \mathrm{~m}$ of the "actual" location of the target. The sensing range, $r_{0}$, is $100 \mathrm{~m}$. The target was assumed to be at $[0,0]$, and the a-priori location of the target was also assumed to be $[0,0]$. The measurement error is assumed to be $30 \%$. Values of $\psi_{0}$ and $\phi_{0}$ required in the above algorithm are specified thus: $\psi_{0}=30^{\circ}, \phi_{0}=625 \mathrm{~m}^{2}$.

We have compared the results obtained using the proposed algorithm with results obtained using other criteria for selecting the $3^{\text {rd }}$ sensor, viz. (a) proximity only, (b) collinearity only, and (c) deviation from ideal direction only (see Table 1). The error in estimated location based on our algorithm is also compared with the error resulting from selecting the "best" possible sensor. The latter is obtained by exhaustively computing the least square error estimate for each possible sensor.

It may be noted that using the proposed algorithm, we achieve near minimum error in estimated location. Further, as $N$ increases, there is improvement in the error in estimated location. However, the error in estimated location does not improve when (a) the $3^{r d}$ sensor is selected based only on the ideal direction since the resulting three sensors may be collinear, in which case the estimation error may be large.

Table 1. Comparison of location estimation error with proposed algorithm and with other algorithms.

\begin{tabular}{cccccc}
\hline $\mathrm{N}$ & \multicolumn{4}{c}{ Collinearity only Proximity only Ideal direction only } & \multicolumn{3}{c}{ Best sensor Proposed algorithm } \\
\hline 10 & 11.7581 & 24.6160 & 15.2932 & 9.1745 & 9.4021 \\
\hline 20 & 11.5150 & 19.4959 & 16.9380 & 8.2882 & 8.7331 \\
\hline 30 & 10.2022 & 13.1000 & 17.4704 & 8.1151 & 8.5329 \\
\hline
\end{tabular}

\section{Conclusion}

In this paper, we have proposed that the sensors be selected based on three measures viz. (a) collinearity, (b) ideal direction in which the sensor should be selected so that 
the error is minimized, and (c) proximity of the sensor from the target. We use measurements that are subject to multiplicative error. Further, we use least square error estimation technique to estimate the target location.

The sensor selection is done by the central 'tracker" and only the selected sensors measure distance to the target and communicate to the central "tracker" for estimating the target location.

We propose that a $3^{\text {rd }}$ sensor be selected in the ideal direction calculated on the basis of given two sensor positions. The knowledge of a-priori target position is assumed to be available. The results obtained with proposed algorithm are very very encouraging.

\section{References}

1. P. Bahl and V. N. Padmanabhan, "RADAR: An in-building RF-based user location and tracking system," in In INFOCOM, TelAviv, Israel, 2000.

2. D. Niculescu and B. Nath, "Ad Hoc Positioning System," in In GLOBECOM, San Antonio, 2001.

3. N.B.Priyantha, A.Chakraborty, and H.Balakrishnan, "The cricket location-support system," in In ACM MOBICOM, Boston, MA, 2000.

4. A.Savvides, C.C.Han, and M.Srivastava, "Dynamic fi ne-grained location-support system," in In ACM MOBICOM, 2001.

5. B. Parkinson and J. Spilker, "Global positioning system: Theory and application," in American Institute of Astronautics and Aeronautics, 1996.

6. F. Zhao, J. Liu, L. Guibas, and J. Reich, "Collaborative signal and information processing: An information directed approach," in Proceedings of the IEEE, vol. 91, no. 8, 2003.

7. J. Liu, J. Reich, and F. Zhao, "Collaborative in-network processing for target tracking," in EURASIP JASP, vol. 4, no. 378-391, 2003.

8. E. Ertin, J. W. Fisher, and L. C. Potter, "Maximum mutual information principle for dynamic sensor query problems," in 2nd International Workshop on Information Processing in Sensor Networks, 2003.

9. H. Wang, K. Yao, G. Pottie, and D. Estrin, "Entropy-based sensor selection heuristic for target localization," in IPSN 2004, Proceedings of the third international symposium on Information processing in sensor networks. ACM Press, 2004.

10. V. Isler and R. Bajcsy, "The sensor selection problem for bounded uncertainty sensing models," in Center for Information Technology Research in the Interest of Society, 2003.

11. M.Betke and L.Gurvits, "Mobile robot localization using landmarks," in Proceedings of the IEEE International Conference on Robotics and Automation, volume 2, pages 135-142, 1994.

12. S.Chits, S.Sundresh, Y.Kwon, and G.Agha, "Cooperative tracking with binary-detection sensor networks," in Technical Report UIUCDCS-R-2003-2379, Computer Science Dept., University of Illinois at Urbana-Champaign, 2003.

13. J. Aslam, Z. Butler, V. Crepi, G. Cybenko, and D. Rus, "Tracking a moving object with a binary sensor network," in ACM International Conference pn Embedded Networked Sensor Systems (SenSys), 2003.

14. Q.X.Wang, W.P.Chen, R.Zheng, K.Lee, and L.Sha, "Acoustic target tracking using tiny wireless sensor devices," in International Workshop on Information Processing in Sensor Networks (ISPN), 2003.

15. W. H. Press, B. P. Flannery, S. A. Teukolsky, and W. T. Vetterling in Numerical Recipes in C, 2002.

16. V. P. Sadaphal and B. N. Jain, "Spread-based heuristics for sensor selection in sensor networks," Technical Report in preparation. 